\title{
WIRELESS SENSOR NETWORKS IN BIOMEDICAL: BODY AREA NETWORKS
}

\author{
Paul Honeine ${ }^{(1)}$, Farah Mourad ${ }^{(1)}$, Maya Kallas $^{(1,2)}$ Hichem Snoussi $^{(1)}$, Hassan Amoud $^{(3)}$, Clovis Francis ${ }^{(2)}$ \\ (1) Institut Charles Delaunay (CNRS), LM2S, Université de Technologie de Troyes, France \\ (2) Laboratoire d'analyse des systèmes (LASYS), Université Libanaise, Lebanon \\ (3) Azm Center for Research in Biotechnology and its Applications, Lebanese University, Lebanon
}

\begin{abstract}
The rapid growth in biomedical sensors, low-power circuits and wireless communications has enabled a new generation of wireless sensor networks: the body area networks. These networks are composed of tiny, cheap and low-power biomedical nodes, mainly dedicated for healthcare monitoring applications. The objective of these applications is to ensure a continuous monitoring of vital parameters of patients, while giving them the freedom of motion and thereby better quality of healthcare. This paper shows a comparison of body area networks to the wireless sensor networks. In particular, it shows how body area networks borrow and enhance ideas from wireless sensor networks. A study of energy consumption and heat absorption problems is developed for illustration.
\end{abstract}

\section{INTRODUCTION}

Nowadays, biomedical sensors are used solely in health monitoring applications, as with the electrocardiograms. In other words, they are not yet integrated into communicating networks. However, with the increase of the vital body signs to be monitored simultaneously, many biomedical sensors need to work together to collect measurements at the same time. Moreover, it is often important to communicate the measured data from a sensing device to others. Thus, it becomes crucial to have a network of communicating biomedical sensors. This would have a radical impact on the quality of life of patients and on their treatment success rates. It could have a wide range of future applications, as well, such as monitoring of cardiovascular diseases, diabetes and asthma, consulting cases via telemedicine or health-care systems, etc.

The Body Area Networks (BANs) have recently emerged as a by-product to do so. They are a new generation of Wireless Sensor Networks (WSNs), suited to monitor human bodies. A BAN is composed of a collection of tiny nodes, equipped with biomedical sensors, motion detectors and wireless communication devices. These nodes collect vital body

This work was partly supported by the Franco-Lebanese CEDRE program. signs and then transmit them in a wireless manner to a central unit where all collected information is processed. Due to their wireless nature, BAN nodes have many advantages, such as omnipresence connectivity, mobility and inter-operability [1]. Some nodes may also be equipped with actuators, such as pacemakers or instruments to store and inject medicines.

In this paper, we review some issues in BANs. We show how BAN borrows and enhances ideas from the WSN literature. More specifically, we study the energy consumption and the heat absorption problems, both problems being tightly related.

\section{FROM WSN TO BAN}

\subsection{Wireless sensor networks}

Recent advances in miniaturization and wireless communication have enabled a new class of tiny powerful computers: Wireless Sensor Networks (WSNs). WSNs consist of largescale, highly distributed and self-organized networks, mainly developed for surveillance and monitoring. They are composed of a large number of tiny and cheap nodes, having computing, sensing and communication capabilities. WSNs were initially meant for military applications such as large battlefield surveillance. In these applications, WSNs were assumed to have cubic-millimeter computers, called smart dust [2]. To the best of our knowledge, the smart dust concept has not been realized. However, the issues and challenges of WSNs pave the way to many new opportunities. It is thus expected to use WSNs in a broad range of applications, including environmental monitoring, animal migration monitoring, security surveillance, industrial control, as well as personal healthcare monitoring, etc.

\subsection{Description of body area networks}

The technology behind the WSNs is still under development. However, the research on WSNs does not deal with the challenges associated with human body ${ }^{1}$ monitoring. For this reason, a new generation of WSNs has emerged: the Body Area

\footnotetext{
${ }^{1}$ Body area networks are not limited to humans.
} 
Table 1. Some differences between WSNs and BANs.

\begin{tabular}{lcc}
\hline & Wireless Sensor Networks & Body Area Networks \\
\hline Scale & Wide area coverage (up to several kilometers) & Limited by the human body (in centimeters) \\
Node number & Huge number of nodes for coverage & Limited number of pervasive nodes \\
Accuracy & Compensated by the redundancy & Accurate measurements are required by each node \\
Failure & Nodes often disposable & Difficult replacement of implanted nodes \\
Energy scavenging & Solar or wind power & Motion or body heat \\
\hline
\end{tabular}

Networks $^{2}$ (BANs). BANs provide a new paradigm for the WSNs technology in the physiological biosensor, the MAC layer, the network layer, the routing strategy, etc. Although WSNs and BANs share many difficulties, such as miniaturization, they face different challenges, as shown in Table 1 [3].

A BAN is a network of wearable on-body computing devices. It can also includes in vivo implanted biosensor devices. According to the IEEE 802.15 (Task Group 6), a BAN consists of "low power devices operating on, in or around the human body (but not limited to humans) to serve a variety of applications including medical, consumer electronics / personal entertainment and other". BANs have a wide range of applications, as, for instance, in gaming or virtual reality. However, the main challenges in terms of research or engineering remain in the biomedical and healthcare monitoring applications. Indeed, evolution of BANs should follow the ever-increasing development in the medical domain; its main objective being to ensure constant vigilant and pervasive monitoring of patients at home or at work.

\subsection{Requirements in body area networks}

Although BANs are essentially WSNs, they take the WSNs to their extreme in many directions. Indeed, BANs for instance are limited to the human body where very few nodes are deployed. Having few sensing devices, data loss in BANs could be significant, as opposed to WSNs, where nodes yield redundant information. Therefore, each node needs to provide accurate measurements, especially for nodes giving vital information, such as electrocardiogram (ECG) measurements. This may require additional measurements to ensure quality-of-service and real-time data delivery, since reliability of measurements is a must in the medical domain. Moreover, BANs often consist of networks with devices performing diverse tasks. Examples of these devices include ECG, blood pressure, pulse oximetry, temperature, respiratory, etc. Such heterogeneous characteristics require different frequency acquisition rates as well as different transmission rates.

On the other hand, some devices of the BAN need to be implanted, in vivo, inside the human body. Implanted biosensors should be biocompatible, robust, as replacement is diffi-

\footnotetext{
${ }^{2}$ BANs are also known as wireless body area networks, or body sensor networks.
}

cult, and with low energy requirements, since energy is hard to supply. In addition, in BAN, nodes are in close proximity to or inside the human body. This may lead to high absorption of the electromagnetic radio-frequency waves, and thus to an increase of the body temperature. According to the safety limits for exposure to radio-frequency energy, communications should be uniformly distributed among all the nodes. Moreover, as the human body is not rigid, frequent changes in nodes positions should be considered in the network topology. For these reasons, a special attention should be paid to the design of the BAN, including the routing strategy.

\section{ENERGY SCAVENGING}

The energy consumption in BANs is crucial, especially in implanted biosensors, since they are inaccessible and difficult to replace. For instance, a pacemaker battery usually lasts for 5 to 10 years. Since the battery is sealed inside the pacemaker, replacing the battery leads to the replacement of the entire system. In order to increase the lifetime of battery-powered devices, extra amount of energy can be delivered by energy harvesting, i.e., energy scavenging [4]. As opposed to WSNs, where a great attention is paid to solar energy, BANs introduced a new nature of energy scavenging. Devices can scavenge power from the human body using body heat and body vibrations. Although heat and motion of the human body bring new opportunities, they also introduce new challenges, as shown in the following.

\subsection{Body motion}

Electrical power can be generated from motion and vibration, with the so-called inertial-power scavenging. Natural human body motion may be easily converted into electrical power. This is illustrated by electronic self-winding watches based on moving mechanics, such as the ETA Autoquartz and the Seiko Automatic Generating System. More promising approaches are based on piezoelectric or capacitive generators. An example of electric power generators is the electric shoes $[5,6]$. For instance, authors of [5] propose electric shoes to provide autonomy to artificial organs.

While motion may provide power to BANs, it introduces new challenges. In fact, as the human body is not rigid, thus the devices can move, at least relatively to each others. Thus, 
frequent changes in the network topology should be considered in the design of the network architecture. BANs should be robust to these changes, for instance by adapting the routing strategy, and the self-organization of the nodes.

\subsection{Body heat}

Temperature difference can be directly converted into electrical power, as given by the Peltier-Seebeck effect. As any heat engine, the efficiency of thermoelectric energy is limited by the Carnot's theorem. In practice, large thermal gradients are required to create satisfactory levels of voltage and power. This limits the application of thermoelectric energy in BANs, provided by body heat over ambient temperature. Nevertheless, recent works are very encouraging [7], as illustrated by the Seiko Thermic watch.

While environment temperature cannot be controlled, human body temperature is essentially ${ }^{3} 37{ }^{\circ} \mathrm{C}\left(98.6{ }^{\circ} \mathrm{F}\right)$. In BANs, the temperature is likely to be different than this common value. In fact, radio-frequency transmission of wireless nodes located on-body or implanted contribute to radiation absorption, which may result into thermal effects. For safety reasons, the radiation absorption should be reduced, by controlling the Specific Absorption Rate (SAR) [8] and using an appropriate routing protocol (see Section 4.2).

\section{ROUTING INFORMATION: FROM WSN TO BAN}

In this section, the routing problem in BANs is tackled with techniques from the WSNs literature. Our aim is to show how BANs borrow and enhance ideas from WSNs. Three directions are presented here, with essentially the same goal: reducing the absorption of power in the human body, which is dissipated as heat. Such a problem is addressed by a clusterbased routing, a temperature-aware routing, and using an onbody node for implanted networks.

\subsection{Routing by clustering}

In WSNs, several properties for routing strategies are desirable: maximize system lifetime by uniform battery usage of nodes, and application to large-scale networks. Such properties are not satisfied by classical network protocols, including the direct transmission, multi-hop, and clustering.

The LEACH (Low Energy Adaptive Clustering Hierarchy) [9] strategy fulfills the desired properties by combining, on the one hand a hierarchical protocol with clusterhead nodes to aggregate and compress the data, and on the other hand a randomized rotation of the clusterhead nodes. In fact, a cluster-based hierarchy allows small communication distances for most nodes, the non-clusterhead nodes [10]. As opposed to conventional clustering strategies, LEACH uses

\footnotetext{
${ }^{3}$ Body temperature depends upon the place at which the measurement is made (slightly higher when taken internally), and fluctuates over the day.
}

rotating election of clusterhead nodes and adapting the corresponding clusters, which allows the energy requirements to be uniformly distributed among all the senors. Moreover, data is compressed at each clusterhead node, accomplishing an additional reduction in the energy dissipation.

Several algorithms were derived for BAN based on LEACH, using a node clustering structure. For instance, the authors of [11] focus on the self-organization of nodes, as opposed to the randomness election in LEACH which may lead to some isolated nodes. In [12], the authors combine, on the one hand clustering to reduce the number of long direct communications, and on the other hand parallel, multi-hop indirect transmissions, the co-called chains. Such combination has significantly better energy efficiency than LEACH, as illustrated in [13] for in-body biomedical sensor networks. It is worth noting that LEACH is optimized for large-scale networks, which is not the case for BAN.

\subsection{Temperature-aware routing}

The Specific Absorption Rate (SAR) is defined as power absorbed per unit mass of the tissue, a measure in determining the amount of power lost due to heat dissipation. In BAN, the SAR should be taken into account, either locally or globally using its average over the entire mass of tissue between the transmitter and the receiver. Because of the SAR consideration, the thermal effects of BAN should be considered in the routing protocol. This can be done by balancing the communication over the nodes, as illustrated above with the LEACH algorithm. Moreover, one may also consider the temperature to select appropriate routing paths.

Temperature-aware routing was initially investigated in [14], by rotating clusterhead nodes based on leadership history and node location. In [15], an (adaptive) least temperature routing is proposed by routing data away from high temperature spots and area. To this end, multi-hop routing is applied, where each node selects its neighboring node with the lowest temperature as its next hop. By selecting the next hop, such approach is not optimized globally. To overcome this drawback, one may consider the least total temperature route, by including the shortest path routing. In [16], node temperature is converted into graph weights and minimum temperature route is obtained using Dijkstra's algorithm.

\subsection{Routing: in vivo implanted networks}

More constraints are imposed by in vivo implanted sensor networks, including restrictions due to absorption of communication power in the tissue (SAR), which is dissipated as heat. For this reason, classical air-as-medium propagation models, where losses are due to freespace wave propagation and multipath fading, are no longer appropriate. In the human body, the saline-water nature of the human tissues contributes considerably to the absorption of the electromagnetic waves in 
communication. There has been considerable research going on in the field of measurement of calculation of SAR in human tissues, and specification of safe absorption rates [17].

It is well known that the in-body propagation loss is much more than that of on-body propagation. Thus, routing data among implanted nodes may be inappropriate for long range communications. In [18], a new network architecture is proposed for the implanted networks by introducing an on-body coordinator. The coordinator plays the role of a beacon, by forwarding the data from one implanted node to another over long distance more safely and efficiently. Since a coordinator is on-body, it may include other functionalities, such as monitoring and more power resources.

\section{FINAL REMARKS}

In this paper, our aim was to show how BAN and WSN are intimately connected, borrowing and enhancing ideas from each others. This was illustrated on the routing problem, where BAN incorporates additional constraints including energy consumption, SAR and temperature considerations. All these constraints are intimately connected. It is obvious that this paper is not a survey on BAN. For instance, the physiological biosensors and the MAC layer were not studied, nor security issues $[19,20]$. We refer the interested reader to more comprehensive surveys $[21,22,23]$. It is worth noting that most of these surveys were published in 2011, subsequent to the initial submission of this work.

\section{REFERENCES}

[1] D. Cypher, N. Chevrollier, N. Montavont, and N. Golmie, "Prevailing over wires in healthcare environments: benefits and challenges," IEEE Communications Magazine, vol. 44, no. 4, pp. 56-63, 2006.

[2] B. Warneke, K. S. J, and S. Dust, "Smart dust: Communicating with a cubic-millimeter computer," Classical Papers on Computational Logic, vol. 1, pp. 372-383, 2001.

[3] G.-Z. Yang, Body Sensor Networks. Secaucus, NJ, USA: SpringerVerlag New York, Inc., 2006.

[4] J. A. Paradiso and T. Starner, "Energy scavenging for mobile and wireless electronics," Pervasive Computing, IEEE, vol. 4, no. 1, pp. 18-27, Mar. 2005.

[5] J. F. Antaki, G. E. Bertocci, E. C. Green, A. Nadeem, T. Rintoul, R. L. Kormos, and B. P. Griffith, "A gait-powered autologous battery charging system for artificial organs." ASAIO J., vol. 41, no. 3, pp. M588-95, 1995.

[6] N. S. Shenck and J. A. Paradiso, "Energy scavenging with shoemounted piezoelectrics," IEEE Micro, vol. 21, pp. 30-42, May 2001.

[7] J. W. Stevens, "Optimal design of small $\Delta \mathrm{T}$ thermoelectric generation systems," Energy Conversion and Management, vol. 42, pp. 709-720, 2001.

[8] H. Ren and M. Meng, "Rate control to reduce bioeffects in wireless biomedical sensor networks," in Mobile and Ubiquitous Systems, Annual International Conference on. Los Alamitos, CA, USA: IEEE Computer Society, July 2006, pp. 1-7.

[9] W. R. Heinzelman, A. Chandrakasan, and H. Balakrishnan, "Energyefficient communication protocol for wireless microsensor networks," in Proceedings of the 33rd Hawaii International Conference on System
Sciences-Volume 8 - Volume 8, ser. HICSS '00. Washington, DC, USA: IEEE Computer Society, 2000.

[10] O. Younis, M. Krunz, and S. Ramasubramanian, "Node clustering in wireless sensor networks: Recent developments and deployment challenges," IEEE Network Magazine, vol. 20, pp. 20-25, 2006.

[11] T. Watteyne, I. Augé-Blum, M. Dohler, and D. Barthel, "Anybody: a self-organization protocol for body area networks," in Proceedings of the ICST 2nd international conference on Body area networks, ser. BodyNets '07. ICST, Brussels, Belgium, Belgium: Institute for Computer Sciences, Social-Informatics and Telecommunications Engineering, 2007, pp. 6:1-6:7.

[12] B. J. Culpepper, L. Dung, and M. Moh, "Design and analysis of hybrid indirect transmissions (hit) for data gathering in wireless micro sensor networks," SIGMOBILE Mob. Comput. Commun. Rev., vol. 8, pp. 6183, January 2004.

[13] M. Moh, B. Culpepper, L. Dung, T.-S. Moh, T. Hamada, and C.-F. Su, "On data gathering protocols for in-body biomedical sensor networks," in IEEE Global Telecommunications Conference (GLOBECOM'05), St. Louis, MO, USA, Dec. 2005.

[14] Q. Tang, N. Tummala, E. K. S. Gupta, L. Schwiebert, S. Member, and S. Member, "Communication scheduling to minimize thermal effects of implanted biosensor networks in homogeneous tissue," IEEE Tran. Biomedical Eng, vol. 52, pp. 1285-1294, 2005.

[15] A. Bag and M. Bassiouni, "Energy efficient thermal aware routing algorithms for embedded biomedical sensor networks," IEEE International Conference on Mobile Adhoc and Sensor Systems Conference, vol. 0, pp. 604-609, 2006.

[16] D. Takahashi, Y. Xiao, F. Hu, J. Chen, and Y. Sun, "Temperature-aware routing for telemedicine applications in embedded biomedical sensor networks," EURASIP J. Wirel. Commun. Netw., vol. 2008, pp. 26:126:26, January 2008.

[17] "A practical guide to the determination of human exposure to radiofrequency fields," NCRP, Tech. Rep. 119, 1993.

[18] B. Zhen, K. Takizawa, T. Aoyagi, and R. Kohno, "A body surface coordinator for implanted biosensor networks," in Proceedings of the 2009 IEEE international conference on Communications, ser. ICC'09. Piscataway, NJ, USA: IEEE Press, 2009, pp. 475-479.

[19] E. Stuart, M. Moh, and T.-S. Moh, "Privacy and security in biomedical applications of wireless sensor networks," in First International Symposium on Applied Sciences on Biomedical and Communication Technologies ISABEL '08, Aalborg, Oct. 2008, pp. 1-5.

[20] L. W. Hanlen, D. Smith, J. A. Zhang, and D. Lewis, "Key-sharing via channel randomness in narrowband body area networks: is everyday movement sufficient?" in Proceedings of the Fourth International Conference on Body Area Networks, ser. BodyNets '09. Brussels, Belgium, Belgium: Institute for Computer Sciences, Social-Informatics and Telecommunications Engineering, 2009, pp. 17:1-17:6.

[21] S. Ullah, H. Higgins, B. Braem, B. Latre, C. Blondia, I. Moerman, S. Saleem, Z. Rahman, and K. Kwak, "A comprehensive survey of wireless body area networks," Journal of Medical Systems, pp. 1-30, Aug. 2010.

[22] M. Chen, S. Gonzalez, A. Vasilakos, H. Cao, and V. Leung, "Body area networks: A survey," Mobile Networks and Applications, vol. 16, no. 2, pp. 171-193, 2011.

[23] B. Latré, B. Braem, I. Moerman, C. Blondia, and P. Demeester, "A survey on wireless body area networks," Wireless Networks, vol. 17, pp. 1-18, January 2011. 